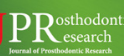 \\ Journal of Prosthodontic Research
}

\author{
Official Journal of Japan Prosthodontic Society
}

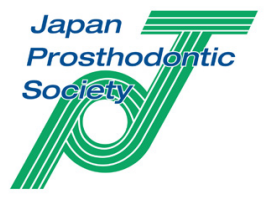

Original article

\section{Patient-reported outcomes of implant-assisted removable partial dentures with magnetic attachments using short implants: A prospective study}

\author{
Masatoshi Negoro ${ }^{a}$, Manabu Kanazawa, ${ }^{\mathrm{a}, *}$, Daisuke Sato ${ }^{\mathrm{b}}$, Ryo Shimada ${ }^{\mathrm{a}}$, Anna Miyayasu ${ }^{\mathrm{a}}$, Mari Asami ${ }^{\mathrm{a}}$, \\ Awutsadaporn Katheng ${ }^{\mathrm{a}}$, Yuriko Kusumoto ${ }^{\mathrm{c}}$, Yuka Abe ${ }^{\mathrm{c}}$, Kazuyoshi Baba ${ }^{\mathrm{c}}$, Shunsuke Minakuchi ${ }^{\mathrm{a}}$ \\ ${ }^{a}$ Gerodontology and Oral Rehabilitation, Graduate School of Medical and Dental Sciences, Tokyo Medical and Dental University, Tokyo, Japan \\ ${ }^{b}$ Department of Implant Dentistry, School of Dentistry, Showa University, Tokyo, Japan \\ 'Department of Prosthodontics, School of Dentistry, Showa University, Tokyo, Japan
}

\begin{abstract}
Purpose: Implant-assisted removable partial dentures (IARPDs) with short implants improve the oral functions of removable partial dentures (RPDs). This study aimed to compare the patient-reported outcomes of RPDs and IARPDs with short implants retained by magnetic attachments.

Methods: We recruited 30 participants with mandibular Kennedy Class I or II and distal extension defects of three or more teeth. RPDs, IARPDs with a healing cap, and IARPDs with a magnetic attachment were evaluated across stages using patient-reported outcomes. All participants completed questionnaires (oral health-related quality of life [OHRQoL], patient general satisfaction, and patient's denture assessment [PDA]) at each stage. The OHRQoL was evaluated using the Oral Health Impact Profile-J 54 (OHIP-J 54) score. The general patient satisfaction was evaluated using a $100 \mathrm{~mm}$ visual analog scale. The Wilcoxon signed-rank test and Bonferroni correction were used to evaluate differences between the groups $(\alpha=0.05)$.

Results: The OHRQoL of IARPDs was significantly higher than that of RPDs. IARPDs with a magnetic attachment had significantly better patient general satisfaction and PDA than IARPDs with a healing cap.

Conclusions: The OHRQoL, patient general satisfaction, and PDA were improved by IARPD with a short implant using a magnetic attachment.

Keywords: Implant-assisted removable partial denture, Oral health quality of life, Short implants, Magnetic attachment, Patient's denture assessment
\end{abstract}

Received 15 September 2020, Accepted 24 January 2021, Available online 1 July 2021

\section{Introduction}

Implant-assisted removable partial dentures (IARPDs) are used to improve various oral functions in patients with removable partial dentures (RPDs) . The IARPD provides a more favorable transfer force by placing the implant in the distal extension defect of the edentulous ridge. [1-3 ] Several studies reported that the oral health-related quality of life (OHRQoL) and patient general satisfaction were significantly higher with IARPDs than with RPDs. [4-8]

It is often difficult to place IARPDs due to a resorption of the remaining edentulous ridge. [9] At present, a technique using short implants has become increasingly popular, as this technique enables safe placement in insufficient bone, thereby avoiding major surgery. [10] Gates et al. performed a prospective study on IARPDs with short implants, in which the OHRQoL using the Oral Health Impact Profile (OHIP)-49, reported that IARPDs provided a higher OHRQoL than conventional RPDs. [11] Jensen et al. conducted a crossover randomized controlled trial (RCT) on 30 participants in Kennedy class I with IARPDs. The results indicated that the IARPD resulted in a higher OHRQoL and patient general satisfaction than the conventional RPD, regardless of the implantation position. [12]

\footnotetext{
* Corresponding author at: Gerodontology and Oral Rehabilitation, Graduate School of Medical and Dental Sciences, Tokyo Medical and Dental University, 5-45 Ochanomizu, Bunkyo-ku, Tokyo 113-8510, Japan.

E-mail address: m.kanazawa.gerd@tmd.ac.jp (M. Kanazawa).
}

https://doi.org/10.2186/jpr.JPR_D_20_00221

1883-1958/C 2020 Japan Prosthodontic Society. All rights reserved.
Cristache et al. conducted a three-group ( $\mathrm{n}=23$ per group) parallel $\mathrm{RCT}$ on mandibular two-implant overdentures using three types of attachments (magnet, locator, and ball) over a 5-year follow-up period. Magnetic attachment requires low maintenance and possesses a high success rate. [13] However, no clinical studies have previously been conducted that compared the healing cap and magnetic attachment in conjunction with IARPDs with short implants.

OHIP and patient general satisfaction have previously been used to determine patient-reported outcomes. [14] Although patient general satisfaction can assess the prosthesis, it has not been possible to evaluate why patient satisfaction improved. $[15,16]$ In addition, although OHIP is effective for OHRQoL evaluation, it is difficult to evaluate positive changes in patients whose baseline OHRQoL did not deteriorate significantly. [17] Therefore, the patient's denture assessment (PDA) has been reported as a new denture evaluation method. [18] PDA is an effective evaluation method for dentures; [19] however, no previous clinical study on IARPDs has investigated patient-reported outcomes using PDA.

The null hypothesis in this prospective clinical study was that there is no difference in OHRQoL, general patient satisfaction, or PDA among RPD, IARPD with a healing cap, and IARPD with a magnetic attachment. The purpose of this study was to compare RPD, IARPD with a healing cap, and IARPD with a magnetic attachment using patient-reported outcomes.

\section{Material and methods}

\subsection{Participants}

This prospective study was performed by comparing conventional RPD, 
IARPD with healing cap, and IARPD with magnetic attachment using a short implant (clinical trial registration: UMIN00002276). The patients recruited had previously visited one of two dental university hospitals located in Japan. This study was conducted in accordance with the guidelines of the Declaration of Helsinki. The Ethics Committee at the Faculty of Dentistry, Tokyo Medical and Dental University (approval number: D2016-026) and the Institutional Review Board of the Showa University Dental Hospital (approval number: DH2016-024) approved this study. All participants were given oral and written information about the study, and they provided written informed consent. Thirty participants met the following inclusion criteria: (1) between 45 and 85 years, regardless of the sex; (2) had mandibular Kennedy Class I or II and a distal extension defect of at least 3 teeth; (3) had one or more teeth with a tooth mobility $\leq 1$ degree, with crowns on the remaining teeth; and (4) sufficient bone volume to receive implants. Patients with the following conditions were excluded from the study: (1) uncontrolled systemic disease; (2) uncontrolled temporomandibular joint arthrosis; (3) dry mouth; (4) infectious disease; (5) oral motor dysfunction; (6) dementia; or (7) uncontrolled psychiatric disorders.

\subsection{Surgical and prosthetic procedures}

Preoperative clinical evaluation was conducted using panoramic radiographs of each mandible. The patient's existing RPD was used if it was deemed clinically appropriate, which was comprehensively assessed by a prosthodontic specialist with more than 10 years of clinical experience. However, a new RPD was fabricated for all patients who did not use an appropriate RPD. Stage 0 was defined using conventional RPDs. All patients underwent intravenous sedation, and all implants were placed by the same implantologist, who was associated with the universities at which the study was conducted. Cone-beam computed tomography was performed, and implant treatment was planned using simulation software (NobelClinician, Nobelbiocare, Zurich, Switzerland). The implants $(6 \times 4.1$ $\mathrm{mm}$ ) (Straumann SLActive Standard Plus RN, Straumann, Switzerland) were placed in the most distal site of the defect using a surgical template with at least $1 \mathrm{~mm}$ of buccal and lingual bone left and $2 \mathrm{~mm}$ of vertical safety margin from the inferior alveolar canal. After implant surgery, the implant was covered with a healing cap. The RPD was relieved to avoid contact with the healing cap and prevent stress on the implant. Six weeks later, the support of the RPD with implants was established by adding acrylic resin to the relief part after verifying the implant condition and settling of the denture base; this was called Stage 1. Four weeks later, the RPD was stabilized by attaching magnetic abutments (Magfit IP I series flat $14 \mathrm{~mm}$ or $30 \mathrm{~mm}, 750 \mathrm{gf}$, Aichi Steel, Aichi, Japan) to the inner surface of the RPD using self-polymerizing resin (Unifast III, GC, Tokyo, Japan); the IARPD and the implant and magnetic attachment provided either retention or support to the RPD. This was called stage 2 . Additionally, the installation of the magnetic attachment was standardized using the product manual and a meeting between the clinicians from each institution.

\subsection{Outcomes}

One month after denture stabilization, the questionnaires described below were administered to all participants. The patient-reported outcomes, including OHIP-J 54, patient general satisfaction, and PDA, were included in the three questionnaires. These questions were used at each stage, one month after the denture was stabilized.

\subsubsection{Oral health-related quality of life}

The OHRQoL was assessed as a primary outcome using the Japanese translation and verification version of the OHIP-J 54. [20] We evaluated the effects on each item 1 month after the evaluation date. Answers were provided on a Likert scale (0-never, 1-hardly ever, 2-occasionally, 3-fairly often, and 4-very often), with total scores ranging from 0 to 216 . Higher scores reflected a more impaired OHRQoL. John et al. assigned each question item to four subdomains: oral function (score range 0-40), orofacial appearance (score range 0-24), psychosocial impact (score range $0-72$ ), and orofacial pain (score range $0-28$ ). [21,22]

\subsubsection{Patient general satisfaction}

The patients used the $100 \mathrm{~mm}$ visual analog scale (VAS) with a range from 0 (completely dissatisfied) to 100 (completely satisfied) to evaluate their general satisfaction with the denture. The VAS question was phrased as "how satisfied are you with your prosthesis?" Therefore, a higher score indicated greater satisfaction.

\subsubsection{Patient's denture assessment}

The effects of denture treatment on patient perceptions and consciousness were evaluated using PDA. This provided a multilateral evaluation, which was different from OHIP. PDA focused on each individual domain rather than the total. We analyzed five domains of the PDA: function, lower denture, expectation, esthetics and speech, and importance. Each question item was measured using a VAS ranging from 0 (completely dissatisfied) to 100 (completely satisfied). Higher scores indicated greater satisfaction with RPD.

\subsection{Statistical analysis}

Appropriate sample size could not be calculated because there have been no previous studies of this nature. Therefore, the number of subjects was preliminarily set in this study. The Wilcoxon signed-rank test and Bonferroni correction were used to evaluate the differences between the groups (stage 0, stage 1, and stage 2). The Mann-Whitney U test was used to analyze differences in Kennedy classes I and II. All statistical analyses were performed using statistical software (IBM SPSS Statistics 22, Chicago, IL, USA) at a significance level of $\alpha=0.05$.

\section{Results}

\subsection{Participant characteristics}

From 2016 to 2019, we recruited 30 participants who met the inclusion criteria. Table 1 presents the characteristics of the participants. Fortyeight implants were placed. After implant placement, three implants were removed from three patients due to a lack of osseointegration. Therefore, we evaluated 27 patients ( 20 patients from one participating university and seven patients from the second; 11 men and 16 women).

\subsection{Oral Health Impact Profile-J 54}

Based on the OHIP-J 54 results, there was a significant improvement in the OHRQoL in stages 1 and 2 compared to stage $0(P=0.042$ and $P=0.012$, respectively). There was no significant difference between stages 1 and 2 $(P=1.00)$ in the four domains of the OHIP, and a significant improvement was observed in oral function from stage 0 to stage $1(P=0.018)$. In other domains, no significant differences were observed between the stages. The results are presented in Table 2 .

\subsection{Patient general satisfaction}

There was a significant improvement in the patient general satisfaction in stage 2 compared with stages 0 and $1(P=0.003$ and $P=0.018$, respectively). No significant difference was found between stages 0 and $1(P=0.153)$. The results are presented in Table 3.

\subsection{Patient's denture assessment}

Concerning PDA, there was a significant improvement in stage 2 compared with stages 0 and 1 in the lower denture $(P=0.009$ and $P=0.045)$. There was no significant difference between stages 0 and $1(P=0.855)$. Regarding the esthetics and speech, a significant improvement was identified in stage 2 compared with stage $1(P=0.042)$. Moreover, there was no significant difference between stages 0 and 1 , or between stages 0 and $2(P=1.00, P=1.00$, respectively). There were no significant differences between any of the stages in the other items. The results are presented in Table 4 . Concerning the Kennedy classification, Kennedy class I showed a significant difference in stage 2 compared to 
Table 1. Characteristics of Patients.

\begin{tabular}{ll}
\hline Characteristics of Patients & \\
\hline All patients (n) & 30 \\
Gender (Male/Female; $n)$ & $13 / 17$ \\
Age (y; mean, SD/range) & $70.8(7.1 / 48-83)$ \\
Number of implants 1/2 (n) & $12 / 18$ \\
Kennedy class I/II & $21 / 9$ \\
Opposing arch & \\
$\quad$ Natural dentition (n) & 9 \\
$\quad$ Removable partial denture (n) & 12 \\
$\quad$ Kennedy class I/II/III/IV) (n) & $(3 / 4 / 1 / 4)$ \\
$\quad$ Full denture (n) & 8 \\
$\quad$ Implant overdenture (n) & 1 \\
\hline
\end{tabular}

Kennedy class II in terms of the lower denture $(P=0.003)$. There were no significant differences between any of the stages in the other items. Moreover, no significant difference was observed in the OHIP and general patient satisfaction at any stage. The results are presented in Table 5 .

\section{Discussion}

In this prospective study, we evaluated IARPDs using short implants with magnetic attachments. Concerning patient-reported outcomes, found significant improvements in the OHRQoL, patient general satisfaction, and PDA with IARPDs using magnetic attachments in comparison with the conventional RPD and IARPDs using healing caps. Therefore, the null hypothesis was partially rejected.

We clarified the effectiveness of IARPDs with short implants for Kennedy Class I and II patients in improving the OHRQoL. These results were similar to those of previous studies. $[6,8,11,12]$ Using an IARPD was also effective in improving the oral function domain of the OHIP. IARPDs improved masticatory function and nutritional status compared to conventional RPDs. [5,23-25] Therefore, we speculated that improvement in masticatory function and feeding status may help improve the OHRQoL. In contrast to previous findings, no difference was found in the OHRQoL between stages 1 and 2. [6] When the OHIP was used in those who do not experience substantial problems with their OHRQoL, a ceiling effect occurs because the answers were concentrated on one point. [26] In addition, John et al. reported that the minimal important difference in the OHIP in dentistry was 6 units (95\% CI, 2-9). [27] In this study, the difference between the median values of stages 0 and 1 was 12 units, demonstrating that the OHRQoL had greatly improved with IARPDs using healing caps. Therefore, the ceiling effect was considered to occur between stages 1 and 2. This ceiling effect may also be responsible for the high OHRQoL reported at stage 0 , when assessing the psychosocial impact and orofacial pain, and the subsequent lack of improvement in the OHRQoL was reflected when using IARPDs. Furthermore, the orofacial appearance did not change between stages because the RPD remained unchanged.

We confirmed that the patient general satisfaction improved by using a magnetic attachment rather than a healing cap. IARPD with a healing cap increases the support for the RPD, but the magnetic attachment allows not only support but also retention of the RPD. Therefore, the stability of the denture was improved by resisting both sinking and lifting. These results were similar to those of previous studies. [6] Contrary to former findings, we found no difference in the patient general satisfaction between stage 0 (conventional RPD) and stage 1 (IARPD with healing cap). [4,5,28] The major complaint when using a partial denture of the lower jaw was that foreign matter, such as food residues, entered the mucosal surface of the RPD base. [23] The IARPD with a healing cap did not improve lifting. Therefore, it was presumed that foreign substances could easily enter the mucosal surface of the RPD denture base.

IARPD with the magnetic attachment was more effective than IARPD with a healing cap in improving PDA in the lower denture, esthetics, and speech domains. The lower denture domain improved because the magnetic attachments increased the stability of the RPD. However, there was no significant change in appearance because the same denture was used at each stage. Furthermore, the pronunciation was greatly affected by the morphology of the upper anterior teeth and palate, which did not change in this study. [29] However, pronunciation was a necessary factor for a long adaptation period. It was possible that the difference in the length of time each denture was worn before the patient filled out the questionnaire in stages 1 and 2 could have affected the result. $[18,30]$ Moreover, the results of the PDA provided greater details on the patient's general satisfaction. The improvement in the lower denture, esthetics, and speech domains may have influenced the improvement in the patient's general satisfaction. Additionally, we clarified that the PDA of the lower denture was significantly higher in Kennedy class I than in Kennedy Class II in IARPD with magnetic attachment. In this study, the RPD for Kennedy Class I covered more multiple missing teeth than the RPD for Kennedy Class II. Therefore, the RPD for Kennedy Class I might have had a greater impact on the patient's evaluation of dentures than Kennedy Class II. However, the results of the three questionnaires did not reveal any significant differences between the number of implants.

This study had some limitations. In this study, the upper jaw condition was not specified. Therefore, it is possible to generalize the results. However, the difficulty of fitting mandibular dentures varies depending on the number of occlusal supports and the condition of the residual teeth in the upper jaw. Therefore, future studies are needed to define the conditions of the upper and lower jaw residual teeth. Furthermore, standardizing treatment when conducting a multi-institutional joint study remains difficult. In this study, implant placement surgery was performed by a single implant specialist. However, denture adjustment was performed at each institution by different prosthetic specialists, each with more than 10 years of clinical experience. Therefore, the operators were different in each facility, which may have affected the results. Due to the short follow-up period in this study, long-term observation results could not be obtained. Therefore, it is necessary to establish a longer follow-up period to confirm a long-term prognosis. Additionally, outcomes that require long-term follow-up are currently being investigated. Although this will be reported in the future, we consider that the average value of marginal bone loss within the range of our current investigation does not affect the OHRQoL. Furthermore, three implants were removed due to a lack of osseointegration. This result is valid compared with those of previous studies. [11] Details on this result will be published in a future paper on the survival rate and marginal bone loss. In this study, IARPD with short implants improved the OHRQoL in patients with Kennedy class I or II edentulous spaces. The results also suggested that the use of magnetic attachments improved patient satisfaction with dentures. However, it remains unclear whether long-term stability can be achieved using this method. Finally, since there have been few clinical studies on IARPDs using short implants with magnetic attachments, we conducted the prospective study to confirm its feasibility. However, in the future, it will be necessary to carry out randomized control trials based on the results of this study.

\section{Conclusion}

Despite the limitations of this study, we found that IARPDs with short implants using magnetic attachments could improve the OHRQoL, patient general satisfaction, and PDA in patients with Kennedy class I and II edentulous spaces. In addition, it was possible to obtain higher patient general satisfaction and PDA results by using IARPDs with magnetic attachments than with IARPDs with a healing cap.

\section{Acknowledgements}

The authors thank KAKENHI Foundation C. for supporting this study (grant Number 16K11586-0). 
Table 2. Oral Health Impact Profile Questionnaire (OHIP-J54).

\begin{tabular}{lcccccc}
\hline & \multicolumn{3}{c}{ Median $[95 \% \mathrm{CI}]$} & \multicolumn{3}{c}{$P$-values } \\
\cline { 2 - 7 } OHIP & Stage 0 & Stage 1 & Stage 2 & Stage 0 vs 1 & Stage 0 vs 2 & Stage 1 vs 2 \\
\hline Summary score & $66[37,78]$ & $54[35,78]$ & $58[32,72]$ & $0.042^{*}$ & $0.012^{*}$ & 1.00 \\
Oral Function & $13[10,19]$ & $12[9,16]$ & $12[10,17]$ & $0.018^{*}$ & 0.063 & 1.00 \\
Orofacial Appearance & $8[6,12]$ & $6[5,11]$ & $6[5,10]$ & 0.774 & 0.174 & 1.00 \\
Psychosocial Impact & $18[3,21]$ & $13[3,18]$ & $16[2,19]$ & 0.075 & 0.390 & 1.00 \\
Orofacial Pain & $7[4,9]$ & $7[4,8]$ & $7[3,8]$ & 0.885 & 0.537 & 1.00 \\
\hline
\end{tabular}

Stage $0=$ Removable Partial Denture; Stage 1 = IARPD with healing cap; Stage 2 = IARPD with magnetic attachment

*significant difference $(P<0.05)$.

Table 3. Patient General Satisfaction.

\begin{tabular}{lcccccc}
\hline & \multicolumn{3}{c}{ Median $[95 \% \mathrm{CI}]$} & \multicolumn{2}{c}{$P$-values } \\
\cline { 2 - 6 } & Stage 0 & Stage 1 & Stage 2 & Stage 0 vs 1 & Stage 0 vs 2 & Stage 1 vs 2 \\
\hline Patient general satisfaction & $82[64,88]$ & $83[77,93.5]$ & $93[81,98]$ & 0.153 & $0.003 *$ & $0.018^{*}$ \\
\hline Stage 0
\end{tabular}

Stage $0=$ Removable Partial Denture; Stage 1 = IARPD with healing cap; Stage 2 = IARPD with magnetic attachment

*significant difference $(P<0.05)$.

Table 4. Patient General Satisfaction.

\begin{tabular}{|c|c|c|c|c|c|c|}
\hline \multirow[b]{2}{*}{ PDA } & \multicolumn{3}{|c|}{ Median $[95 \% \mathrm{CI}]$} & \multicolumn{3}{|c|}{$P$-values } \\
\hline & Stage 0 & Stage 1 & Stage 2 & Stage 0 vs 1 & Stage 0 vs 2 & Stage 1 vs 2 \\
\hline Function & $88[77,97]$ & $93[77,98]$ & $96.5[82,100]$ & 0.12 & 1.00 & 0.144 \\
\hline Lower denture & $66.8[47,87]$ & $74.5[52,85]$ & $79.5[74,87]$ & 0.855 & $0.009 *$ & $0.045^{*}$ \\
\hline Exception & $85.3[80,97]$ & $92[82,100]$ & $94.7[86,99]$ & 0.615 & 0.576 & 1.00 \\
\hline Aesthetics \& speech & $85[57,95]$ & $86[61,93]$ & $89.3[71,100]$ & 1.00 & 0.051 & $0.042 *$ \\
\hline Importance & $88[77,96]$ & $90.8[76,97]$ & $85.8[80,94]$ & 0.291 & 0.537 & 1.00 \\
\hline
\end{tabular}

Stage $0=$ Removable Partial Denture; Stage $1=$ IARPD with healing cap; Stage $2=$ IARPD with magnetic attachment

*significant difference $(P<0.05)$

Table 5. Patient General Satisfaction.

\begin{tabular}{|c|c|c|c|c|c|c|c|c|c|}
\hline \multirow[b]{3}{*}{ Kennedy class I/II } & \multicolumn{6}{|c|}{ Median $[95 \% \mathrm{CI}]$} & \multicolumn{3}{|c|}{$P$-values } \\
\hline & \multicolumn{2}{|c|}{ Stage 0} & \multicolumn{2}{|c|}{ Stage 1} & \multicolumn{2}{|c|}{ Stage 2} & \multirow{2}{*}{ Stage 0} & \multirow{2}{*}{ Stage 1} & \multirow{2}{*}{ Stage 2} \\
\hline & $\mathrm{I}$ & II & $\mathrm{I}$ & II & $\mathrm{I}$ & II & & & \\
\hline Function & $87[77,97]$ & $90[81,96]$ & $87[77,97]$ & $97[93,100]$ & $96[83,100]$ & $97[75,98]$ & 0.60 & 0.12 & 0.67 \\
\hline Lower denture & $68[52,87]$ & $67[24,86]$ & $76[50,85]$ & $73[61,77]$ & $84[76,92]$ & $74[68,80]$ & 0.50 & 0.86 & $0.03 *$ \\
\hline Exception & $87[77,98]$ & $93[85,94]$ & $94[75,100]$ & $87[87,100]$ & $95[82,99]$ & $95[94,99]$ & 0.60 & 0.56 & 0.50 \\
\hline Aesthetics \& speech & $83[58,93]$ & $85[34,97]$ & $87[68,92]$ & $86[50,93]$ & $91[77,98]$ & $70[60,100]$ & 0.90 & 0.78 & 0.67 \\
\hline Importance & $86[75,96]$ & $88[83,95]$ & $87[74,96]$ & $94[80,98]$ & $85[80,93]$ & $87[80,98]$ & 0.82 & 0.38 & 0.71 \\
\hline
\end{tabular}

Stage $0=$ Removable Partial Denture; Stage $1=$ IARPD with healing cap; Stage 2 = IARPD with magnetic attachment

*significant difference $(P<0.05)$.

\section{Conflict of interest statement}

All authors report no conflict of interest.

\section{References}

[1] Shahmiri RA, Atieh MA. Mandibular Kennedy Class I implant-tooth-borne removable partial denture: a systematic review. J Oral Rehabil 2010;37:225-34. https://doi.org/10.1111/j.1365-2842.2009.02044.x.

[2] de Freitas RF, de Carvalho Dias K, da Fonte Porto Carreiro A, Barbosa GA, Ferreira MA. Mandibular implant-supported removable partial denture with distal extension: a systematic review. J Oral Rehabil 2012;39:791-8. https://doi. org/10.1111/j.1365-2842.2012.02326.x.

[3] Zancopé K, Abrão GM, Karam FK, Neves FD. Placement of a distal implan to convert a mandibular removable Kennedy class I to an implant-supported partial removable Class III dental prosthesis: a systematic review. J Prosthet Dent 2015;113:528-33. https://doi.org/10.1016/j.prosdent.2014.12.011.
[4] Mitrani R, Brudvik JS, Phillips KM. Posterior implants for distal extension removable prostheses: a retrospective study. Int J Periodontics Restorative Dent 2003;23:353-9. https://doi.org/10.1016/j.prosdent.2003.10.020.

[5] Ohkubo C, Kobayashi M, Suzuki Y, Hosoi T Effect of implant support on distalextension removable partial dentures: in vivo assessment. Int J Oral Maxillofac Implants 2008;23:1095-101. https://doi.org/10.1111/j.1365-2842.2006.01641.x.

[6] Wismeijer D, Tawse-Smith A, Payne AG. Multicentre prospective evaluation of implant-assisted mandibular bilateral distal extension removable partial dentures: patient satisfaction. Clin Oral Implants Res 2013;24:20-7. https://doi. org/10.1111/j.1600-0501.2011.02367.x.

[7] Goncalves TM, Campos CH, Garcia RC. Implant retention and support for distal extension partial removable dental prostheses: satisfaction outcomes. J Prosthet Dent 2014;112:334-9. https://doi.org/10.1016/j.prosdent.2013.11.004.

[8] Campos CH, Gonçalves TM, Garcia RC. Implant-supported removable partial denture improves the quality of life of patients with extreme tooth loss. Braz Dent J 2015;26:463-7. http://dx.doi.org/10.1590/0103-6440201300097.

[9] Esposito M, Grusovin MG, Felice P, Karatzopoulos G, Worthington HV, Coulthard P. Interventions for replacing missing teeth: horizontal and vertical bone augmentation techniques for dental implant treatment. Cochrane Database Syst Rev 2009;2009:CD003607. https://doi.org/10.1002/14651858.CD003607. pub4. 
[10] Thoma DS, Cha JK, Jung UW. Treatment concepts for the posterior maxilla and mandible: short implants versus long implants in augmented bone. J Periodontal Implant Sci 2017;47:2-12. https://doi.org/10.5051/jpis.2017.47.1.2.

[11] Gates WD III, Cooper LF, Sanders AE, Reside GJ, De Kok IJ. The effect of implant-supported removable partial dentures on oral health quality of life. Clin Oral Implants Res 2014;25:207-13. https://doi.org/10.1111/clr.12085.

[12] Jensen C, Raghoebar GM, Kerdijk W, Meijer HJ, Cune MS. Implant-supported mandibular removable partial dentures; patient-based outcome measures in relation to implant position. J Dent 2016;55:92-8. https://doi.org/10.1016/ i.jdent.2016.10.008.

[13] Cristache CM, Muntianu LA, Burlibasa M, Didilescu AC. Five-year clinica trial using three attachment systems for implant overdentures. Clin Oral Implants Res 2014;25:e171-e178. https://doi.org/10.1111/clr.12086.

[14] Allen PF, McMillan AS, Walshaw D, Locker D. A comparison of the validity of generic- and disease-specific measures in the assessment of oral health-related quality of life. Community Dent Oral Epidemiol 1999;27:344-52. https://doi. org/10.1111/j.1600-0528.1999.tb02031.x.

[15] Divaris K, Ntounis A, Marinis A, Polyzois GL, Polychronopoulou A. Patients' profiles and perceptions of complete dentures in a university dental clinic. Int $\mathrm{J}$ Prosthodont 2012;25:145-7.

[16] Stober T, Danner D, Lehmann F, Séché AC, Rammelsberg P, Hassel AJ. Association between patient satisfaction with complete dentures and oral healthrelated quality of life: two-year longitudinal assessment. Clin Oral Investig 2012;16:313-8. https://doi.org/10.1007/s00784-010-0483-x.

[17] McGrath C, Broder H, Wilson-Genderson M. Assessing the impact of oral health on the life quality of children: implications for research and practice. Community Dent Oral Epidemiol 2004;32:81-5. https://doi.org/10.1111/j.16000528.2004.00149.X.

[18] Komagamine Y, Kanazawa M, Kaiba Y, Sato Y, Minakuchi S, Sasaki Y. Association between self-assessment of complete dentures and oral healthrelated quality of life. J Oral Rehabil 2012;39:847-57. https://doi.org/10.1111/ joor. 12004

[19] Komagamine Y, Kanazawa M, Kaiba Y, Sato Y, Minakuchi S. Reliability and validity of a questionnaire for self-assessment of complete dentures. BMC Oral Health 2014;14:45. https://doi.org/10.1186/1472-6831-14-45.

[20] Yamazaki M, Inukai M, Baba K, John MT. Japanese version of the Oral Health Impact Profile (OHIP-J). J Oral Rehabil 2007;34:159-68. https://doi. org/10.1111/j.1365-2842.2006.01693.x.
[21] John MT, Reissmann DR, Feuerstahler L, Waller N, Baba K, Larsson P, et al. Exploratory factor analysis of the Oral Health Impact Profile. J Oral Rehabil 2014;41:635-43. https://doi.org/10.1111/joor.12192.

[22] John MT, Feuerstahler L, Waller N, Baba K, Larsson P, Celebić A, et al. Confirmatory factor analysis of the Oral Health Impact Profile. J Oral Rehabil 2014;41:644-52. https://doi.org/10.1111/joor.12191.

[23] Campos CH, Gonçalves TM, Rodrigues Garcia RC. Implant retainers for freeend removable partial dentures affect mastication and nutrient intake. Clin Oral Implants Res 2014;25:957-61. https://doi.org/10.1111/clr.12165.

[24] Gonçalves TM, Campos CH, Rodrigues Garcia RC. Mastication and jaw motion of partially edentulous patients are affected by different implant-based prostheses. J Oral Rehabil 2014;41:507-14. https://doi.org/10.1111/ioor.12176.

[25] Jensen C, Speksnijder CM, Raghoebar GM, Kerdijk W, Meijer HJA, Cune MS. Implant-supported mandibular removable partial dentures: functional, clinical and radiographical parameters in relation to implant position. Clin Implant Dent Relat Res 2017;19:432-9. https://doi.org/10.1111/cid.12484.

[26] Brennan DS, Spencer AJ. Mapping oral health related quality of life to generic health state values. BMC Health Serv Res 2006;6:96. https://doi. org/10.1186/1472-6963-6-96.

[27] John MT, Reissmann DR, Szentpétery A, Steele J. An approach to define clinical significance in prosthodontics. J Prosthodont 2009;18:455-60. https:// doi.org/10.1111/j.1532-849X.2009.00457.X

[28] Suzuki Y, Kono K, Shimpo H, Sato Y, Ohkubo C. Clinical evaluation of implant-supported removable partial dentures with a stress-breaking attachment. Implant Dent 2017;26:516-23. https://doi.org/10.1097/id.0000000000000592.

[29] Broka K, Vidzis A, Grigorjevs J, Sokolovs J, Zigurs G. The influence of the design of removable dentures on patient's voice quality. Stomatologija $2013 ; 15: 20-5$

[30] Knipfer C, Riemann M, Bocklet T, Noeth E, Schuster M, Sokol B, et al. Speech intelligibility enhancement after maxillary denture treatment and its impact on quality of life. Int J Prosthodont 2014;27:61-9. https://doi.org/10.11607/ ijp.3597.

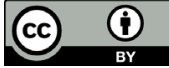

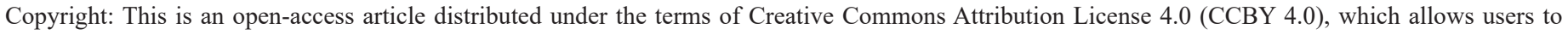
distribute and copy the material in any format so long as attribution is given to the author(s). 